\title{
NOTE
}

\section{Evaluation of 3D Printer Accuracy in Producing Fractal Structure}

\author{
Kana Kikegawa', Kyuuichirou Takamatsu², Masaru Kawakami², Hidemitsu Furukawa², \\ Hiroyuki Mayama ${ }^{3}$ and Yoshimune Nonomura ${ }^{1 *}$ \\ ${ }^{1}$ Department of Biochemical Engineering, Graduate School of Science and Engineering, Yamagata University (4-3-16 Jonan, Yonezawa \\ 992-8510, JAPAN) \\ ${ }^{2}$ Department of Mechanical Systems Engineering, Graduate School of Science and Engineering, Yamagata University (4-3-16 Jonan, Yonezawa \\ 992-8510, JAPAN) \\ ${ }^{3}$ Department of Chemistry, Asahikawa Medical University (2-1-1-1 Midorigaoka-Higashi, Asahikawa, Hokkaido 078-8510, JAPAN)
}

\begin{abstract}
Hierarchical structures, also known as fractal structures, exhibit advantageous material properties, such as water- and oil-repellency as well as other useful optical characteristics, owing to its selfsimilarity. Various methods have been developed for producing hierarchical geometrical structures. Recently, fractal structures have been manufactured using a 3D printing technique that involves computeraided design data. In this study, we confirmed the accuracy of geometrical structures when Koch curve-like fractal structures with zero to three generations were printed using a 3D printer. The fractal dimension was analyzed using a box-counting method. This analysis indicated that the fractal dimension of the third generation hierarchical structure was approximately the same as that of the ideal Koch curve. These findings demonstrate that the design and production of fractal structures can be controlled using a 3D printer. Although the interior angle deviated from the ideal value, the side length could be precisely controlled.
\end{abstract}

Key words: 3D printer, 3D printer accuracy, fractal structure, Koch curve, geometric structure

\section{INTRODUCTION}

Hierarchical structures comprising fat or resin materials exhibit excellent interfacial properties. For example, the lotus leaf exhibits super water-repellency as the fine papillae on the leaf surface are covered with hydrophobic wax crystals on the order of submicrons ${ }^{1-5)}$. Furthermore, the hierarchical structure allows water droplets to act as selfcleaning agents ${ }^{1)}$. Super oil-repellent surfaces have also been developed. For instance, the contact angle of rapeseed oil on a fluorinated rough aluminum surface was reported to be greater than $150^{\circ 6,7)}$. Another useful application is localizing electromagnetic waves in a 3D fractal structure of several alloys comprising $\mathrm{Bi}-\mathrm{Pb}-\mathrm{Sn}$ or epoxy resin materials incorporated with $\mathrm{TiO}_{2}-\mathrm{SiO}_{2}$ composite particles $^{8-10)}$. Fractal structures can also be applied to electrode materials. The emission intensity of fluorescein-labeled human serum albumin was enhanced when fractal silver structures were formed on or near the silver elec- $\operatorname{trodes}^{11)}$.

Various methods have been developed for producing hierarchical geometrical structures ${ }^{12-16}$. Alkyl ketene dimer (AKD) spontaneously forms fractal structures of several microns when solidified from the melted state. The contact angle of the water droplet on the AKD surface is $174^{\circ}$, indicating its super water-repellency. Porous fractal structures on the order of several tens of microns to several hundreds of nanometers were obtained by the anodic oxidization of an aluminum plate. Super oil-repellent surfaces were subsequently manufactured by coating fluorinated monoalkyl phosphate on the fractal surfaces ${ }^{6,7)}$. Artificial formation methods have also been explored. For example, Menger sponge-like fractal structures were produced using a template method ${ }^{17}$ 18). Initially, the template particles were stacked with a suitable density. Then, porous materials were obtained by solidifying the ingredients in the space between the stacked particles. The fractal dimension

\footnotetext{
* Correspondence to: Yoshimune Nonomura, Department of Biochemical Engineering, Graduate School of Science and Engineering, Yamagata University, 4-3-16 Jonan, Yonezawa 992-8510, JAPAN

E-mail: nonoy@yz.yamagata-u.ac.jp

Accepted October 13, 2016 (received for review July 28, 2016)

Journal of Oleo Science ISSN 1345-8957 print / ISSN 1347-3352 online

http://www.jstage.jst.go.jp/browse/jos/ http://mc.manusriptcentral.com/jjocs
} 
of the cross-section of the produced material, pore size distribution, and porosity were found to be closer to those of a Menger sponge. Dendrimers, which are artificially synthesized molecules built up from branched units, contain fractal structures at the molecular scale ${ }^{19-21)}$. Extended molecular fractals, known as "Sierpinski gasket-like structures," were produced with small molecules through selfassembling processes ${ }^{22,23)}$. However, preparing controlled fractal structures is not possible using these methods because many hierarchical structures contain nested geometry and hidden holes.

Recently, fractal structures have been produced using a $3 \mathrm{D}$ printing technique that involves computer-aided design (CAD) data. A plastic artist, Jérémie Brunet, created 3D fractal structures, such as "Sierpinski Octostar," "Fractal Fullerene," and "Miniature fractal building," using a 3D printer $^{24)}$. Although many artists have utilized 3D printers in their creative activities, only a few focus on the accuracy of the shape of the printed objects.

In the present study, we examine the accuracy of the resultant geometrical structures when fractal structures comprising resin materials are printed using a sophisticated 3D printer. We design and print resin models containing Koch curves using a stereolithography 3D printer. This curve exhibits a typical fractal structure with hierarchical triangular shapes shown in Table 1. We examine the printed models to analyze their length, angle, and fractal dimensions using a box-counting method. The fractal dimension is a geometrical parameter indicating the complexity and spatial expanse of hierarchical shapes.

\section{EXPERIMENTAL METHODOLOGY}

\subsection{Design of fractal structures}

The fractal structures were designed using a 3D CAD system, AUTODESK 123D (Autodesk INC, California, USA). The 3D CAD data was converted to a STL file to be exported to a 3D printer. As a representative fractal pattern, the Koch curve was printed on the model surface, as shown in Table 1. The Koch curve is drawn by dividing a straight line into three equal segments and replacing the middle segment with two sides of an equilateral triangle of the same length (the first generation). The process is then repeated for each of the four resulting segments in order to obtain the second and third generations ${ }^{25)}$. The zerothgeneration model is a cuboid $(3 \times 39 \times 14 \mathrm{~mm})$ with a flat surface. In the first-generation model, six regular triangular prisms with a side length of $3 \mathrm{~mm}$ are arranged in $3 \mathrm{~mm}$ intervals on a rectangular surface $(14 \times 39 \mathrm{~mm})$. In the second- and third-generation models, one side is divided into three equal parts in order to replace the middle segments with an equilateral triangle of the same length. The side lengths of the second- and third- generation models are $1 \mathrm{~mm}$ and $0.33 \mathrm{~mm}$, respectively.

\subsection{Preparation of fractal structures}

The fractal structures were prepared using a stereolithography 3D printer (ACCULAS®BA-30H, D-MEC Corporation, Tokyo, Japan). Four types of models were produced in four sets in order to check the accuracy of the printer. The specifications of the apparatus used were as follows: modeling range $=300 \times 300 \times 300 \mu \mathrm{m}$, laser spot diameter $=30-300 \mu \mathrm{m}$, and layer pitch $=30-150 \mu \mathrm{m}$. Figure 1 shows the schematic of the 3D printer. The stereolithog-

Table 1 The images of all of the samples used and the enlarged cross-sections of the prepared models.

Generation $\begin{gathered}\text { The illustration of } \\ \text { the Koch curve }\end{gathered}$ Front view




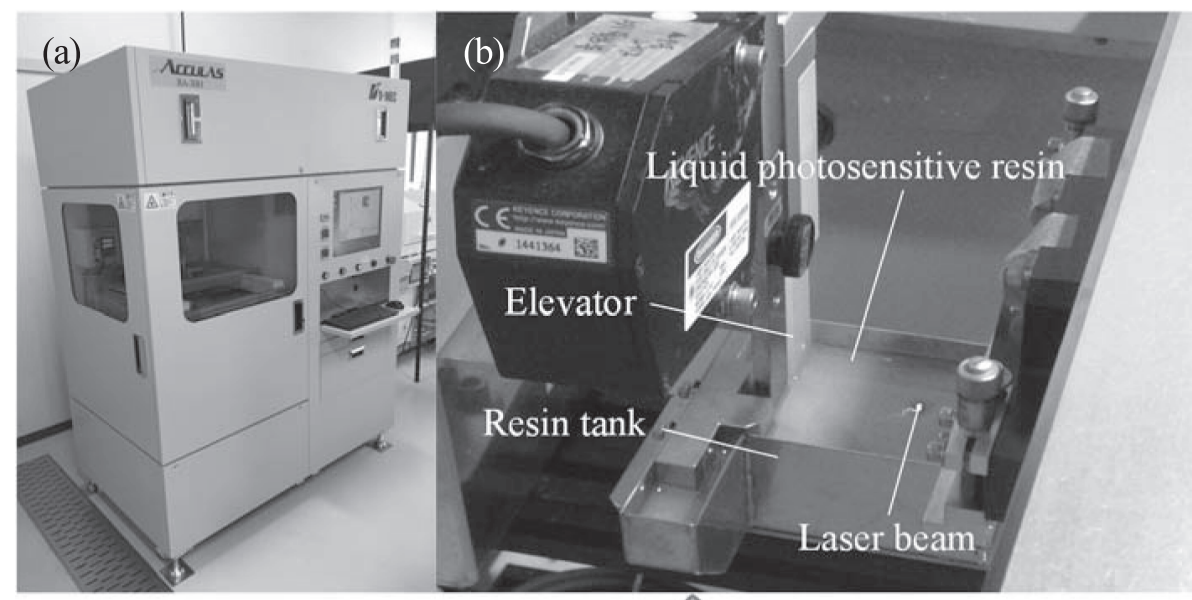

(c)

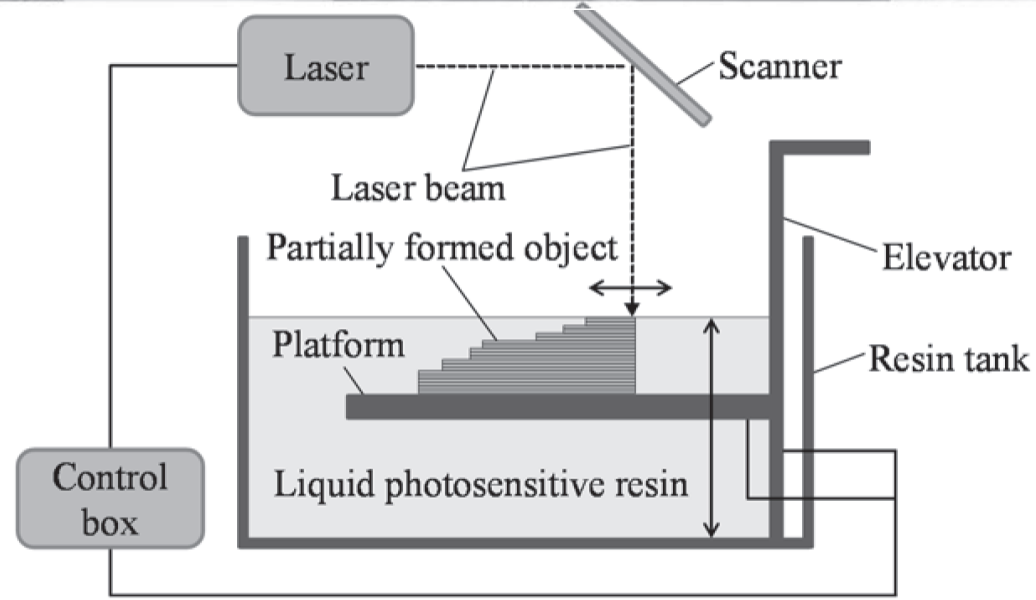

Fig. 1 Photographs of (a) stereolithography 3D printer and (b) model preparation setup. (c) Conceptual diagram of stereolithography 3D printer.

raphy $3 \mathrm{D}$ printer consists of a resin tank filled with a photosensitive liquid, oxetane resin, a movable elevator, a platform, a laser source, and a scanner to control the laser beam. Photosensitive liquid resin was hardened by irradiation with a UV laser along the cross-section of the model surface $^{26,27)}$. After laser irradiation, the elevator moved the hardened resin surface below the liquid surface in order to harden the next cross-section pattern. The production settings used were as follows: laser spot diameter $=60 \mu \mathrm{m}$ and layer pitch $=30 \mu \mathrm{m}$. The obtained model was washed for 4 min in a Branson ultrasonic washer (CPX3800H-J, Japan Emason Corporation, Kanagawa, Japan) filled with the cleaning fluid Solfit AC (KURARAY Corporation, Tokyo, Japan). The extra resin present on the model surface was removed using ethanol and an air duster (AZ Corporation, Osaka, Japan).

\subsection{Measurements}

The cross-section images at 100-fold magnification were captured using wireless digital microscopes (3R-WM401PC and 3R-WM601PC, 3R system Corporation, Fukuoka, Japan). The cross-section was analyzed using the box- counting method in order to evaluate the fractal dimension, $D$. In the box-counting method, an object is first covered with a tetragonal lattice of side $r$. Then, the number of lattices, $N(r)$, that encompass a part of the object is counted $^{28,29)}$. Based on the size of the triangles, we determined the size of $r$ to be in the range of $0.28-5.3 \mathrm{~mm}$, $0.80-3.3 \mathrm{~mm}, 0.85-3.5 \mathrm{~mm}$, and $0.20-3.5 \mathrm{~mm}$ for the zeroth-, first-, second-, and third-generation models, respectively. The box-counting method was performed using the box-counting specialized software, Box counting program ver. 0.1 .

\section{RESULTS AND DISCUSSION}

Table 1 shows images of all of the samples used and the enlarged cross-sections of the prepared models. We succeeded in printing Koch curve-like hierarchical structures on the model surfaces. The ideal curves and cross-sections of the prepared samples are demonstrated in Fig. 2. The deviations of the surface morphology from the theoretical curves were on the order of several tens of micrometers. 
(a)

(c)
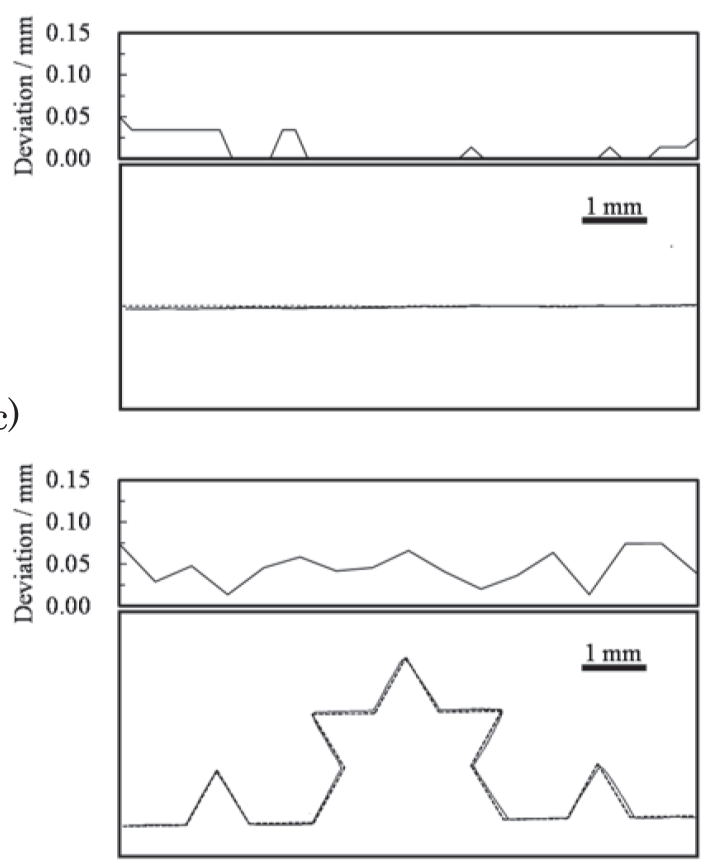

(b)

(d)
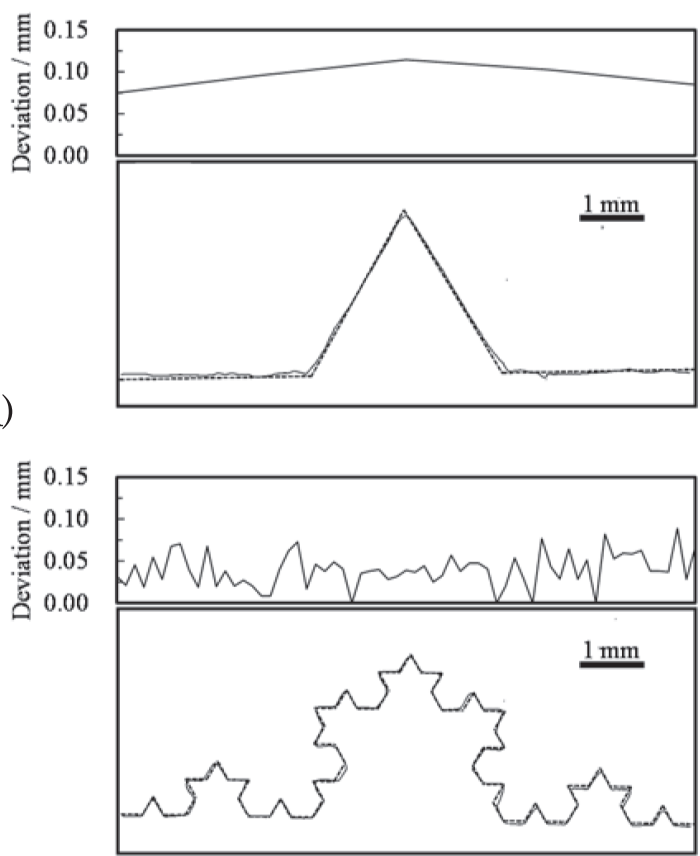

Fig. 2 The cross-section images of prepared samples (straight lines) and the ideal Koch curves (dashed lines) for zeroth-(a), first-(b), second-(c), and third- generation models (d). The deviation of the prepared samples from the theoretical Koch curves were added above the images.

(a)

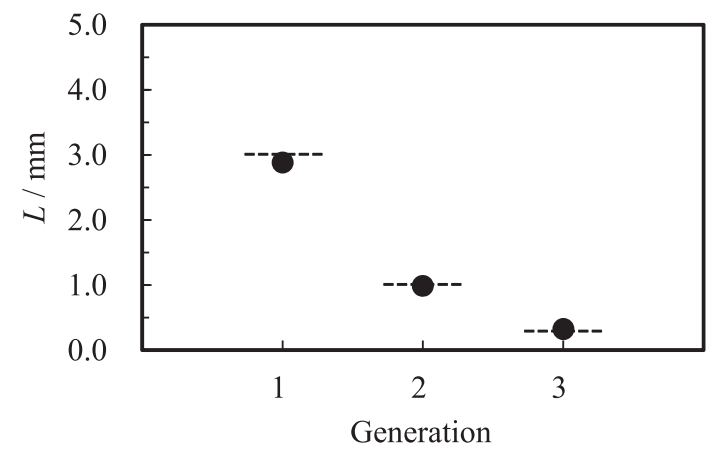

(b)

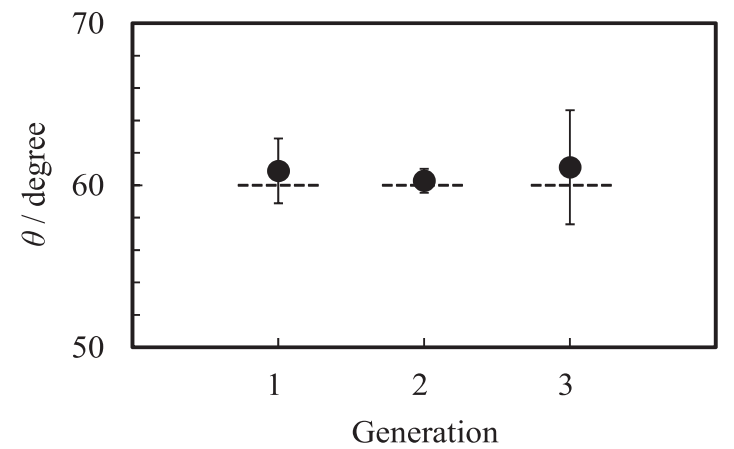

Fig. 3 Geometrical parameters of fractal models: (a) side length and (b) angle.

The side length and interior angles of the triangular structures are shown in Fig. 3. The side lengths of the first-, second-, and third-generation models are $2.89 \pm 0.09 \mathrm{~mm}$, $0.99 \pm 0.04 \mathrm{~mm}$, and $0.33 \pm 0.03 \mathrm{~mm}$, respectively. When the generation number is increased by one, the side length is decreased to one-third of the original length. The interior angles associated with the triangular region of the models are $61 \pm 2^{\circ}, 60 \pm 1^{\circ}$, and $61 \pm 4^{\circ}$ for the first-, second-, and third-generation models, respectively. The third-generation model exhibits the least hierarchical structure in the range of several hundreds of micrometers. Although the side length values are accurate, the interior angle deviated from the ideal value of $60^{\circ}$. The deviation of angles is caused by the rounded shape of the triangular area of the model. The low accuracy of the structure could be attributed to the large laser spot size of $60 \mu \mathrm{m}$, which is too coarse for preparing fine structures in the size of several hundred micrometers.

We evaluated the cross sections to obtain the fractal dimension, $D$, using the box-counting method. The self-similarity is related to the fractal dimension shown as follows ${ }^{30)}$ :

$$
N(r) \propto r^{-D},
$$

where $r$ is the box size, $N(r)$ is the number of boxes required to cover the object, and $D$ is the fractal dimension of the object. As it is difficult to directly measure the fractal dimension $(2<D<3)$, we first measured the fractal dimension of the cross-section $D_{\text {cross }}\left(1<D_{\text {cross }}<2\right)$ using the 
(a)

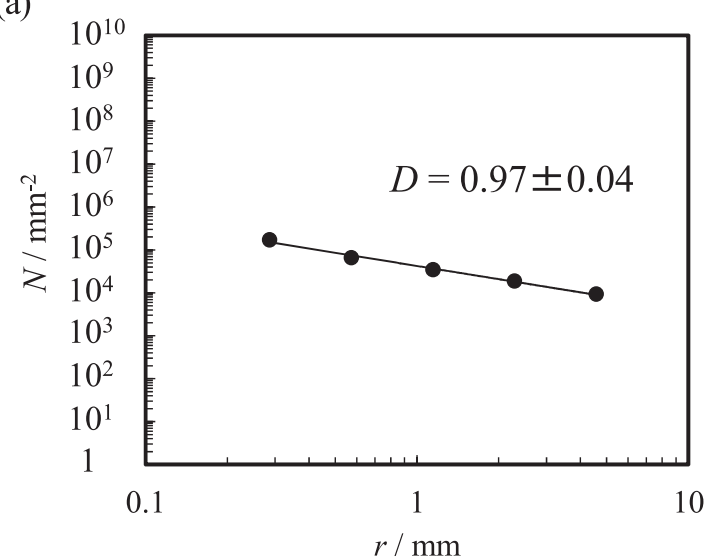

(c)

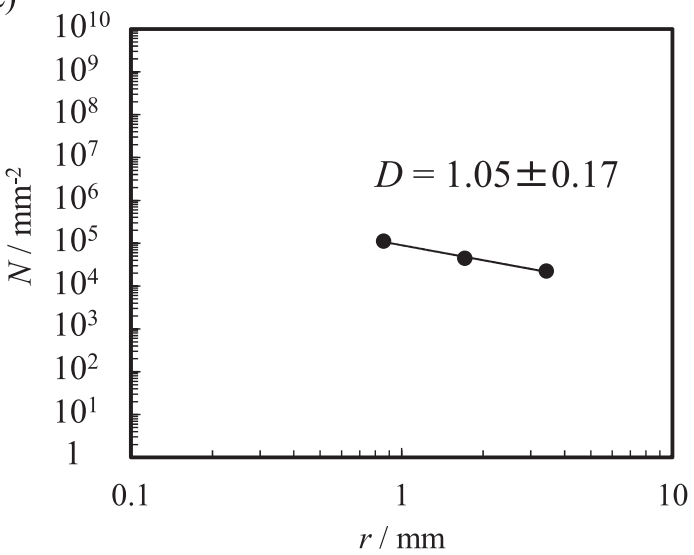

(b)

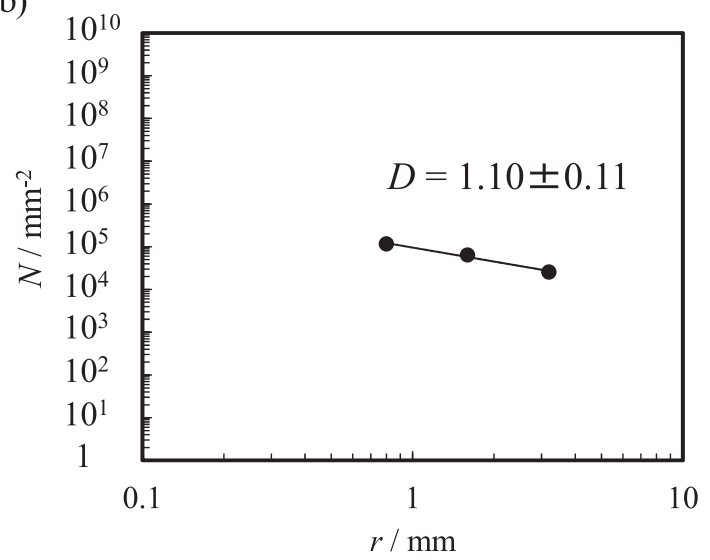

(d)

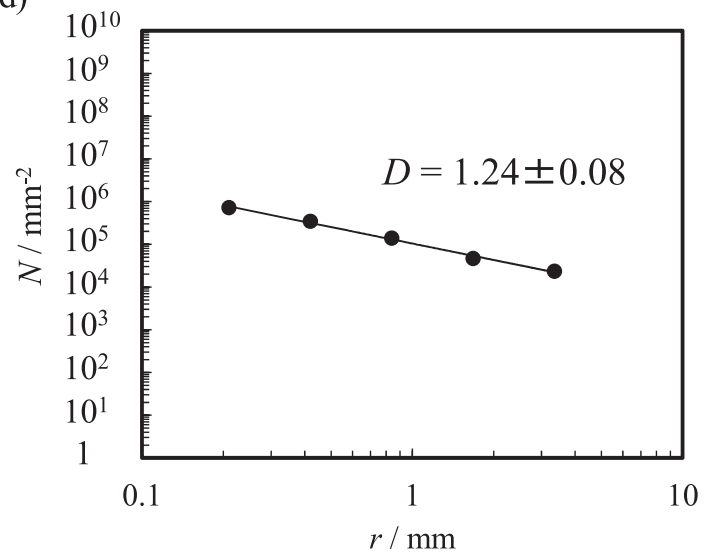

Fig. 4 Number of boxes to cover the object, $N(r)$, as a function of the sizes, $r$ for (a) zeroth Koch curve model, (b) first Koch curve model, (c) second Koch curve model, and (d) third Koch curve model.

box-counting method. The fractal dimension of the surface was then evaluated using the equation $D=D_{\text {cross }}+1$. Figure 4 shows the plot of $\log N(r)$ versus $\log r$ obtained from the digital box-counting data for the zeroth- to third-generation models. The slopes of the straight lines provide the fractal dimensions of the surfaces. The fractal dimensions $\left(D_{\text {cross }}\right)$ of the cross-sections are $0.97 \pm 0.04,1.10 \pm 0.11$, $1.05 \pm 0.17$, and $1.24 \pm 0.08$ for the zeroth-, first-, second-, and third-generation models, respectively, as shown in Fig. 5 . The value of $D_{\text {cross }}$ is nearly equal to one, indicating that the geometrical structure is not hierarchical for the zeroth, first-, and second-generation models. The value of $D_{\text {cross }}$ for the third-generation model, which is approximately equal to the fractal dimension of an ideal $\operatorname{Koch}$ curve $(D=$ 1.26), is larger than that of a flat surface ${ }^{31}$.

As mentioned above, the obtained $D_{\text {cross }}$ value depends on the generation number. In the case of the third-generation model, the obtained dimension was almost identical to that of the ideal Koch curve $(D=1.26)$. The results for the zeroth- and first-generation models also agree with the theory of fractal science because these geometries do not contain any hierarchical structures. On the other hand, we obtained unexpected results from the second-generation

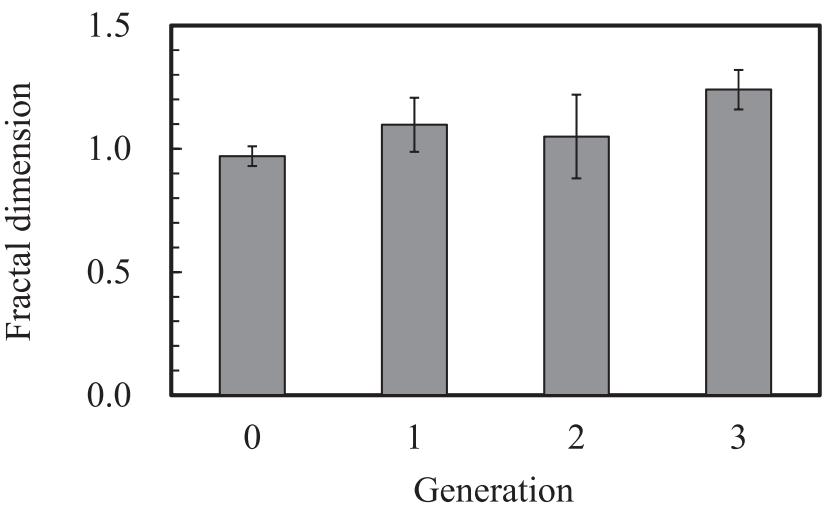

Fig. 5 The fractal dimension, $D$, of fractal models.

model as a nonhierarchical structure was achieved. We hypothesize that this inconsistency is caused by the narrow range of the box size, which contains the hierarchical structure. Previous analysis showed that the ideal fractal dimension is obtained only when the box size range corresponds to the length between the largest and smallest length of triangles in the case of the Koch snowflake ${ }^{32}$. The accurate fractal dimension of the second-generation model was not obtained because the suitable box size range for 


\section{K. Kikegawa, K. Takamatsu and M. Kawakami et al.}

this case would be $1 \mathrm{~mm}$ to $3 \mathrm{~mm}$, which is too narrow for the present analytical method.

\section{CONCLUSIONS}

We printed Koch curve-like fractal structures using a sophisticated 3D printer and evaluated their accuracy. The third-generation model contained hierarchical structures on the order of several hundreds of micrometers. Although the side length was nearly identical to that of the original design, the interior angles deviated from the ideal values because the laser spot size was too large for printing an accurate angle. When analyzing the fractal dimension, the third-generation models showed a hierarchical structure whose fractal dimension was approximately the same as that of the Koch curve $(D=1.26)$. Thus, a sophisticated 3D printer has the potential to build various fractal structures. However, this method is not sufficiently accurate for constructing structures on the order of several tens of micrometers. Commercial 3D printers are often used to manufacture objects such as commodities, action figures, and accessories. However, the accuracy of such 3D printers must be improved in order to successfully use them for applications in the automotive, information, or medical industries. In the future, 3D printers could also be used for preparing novel functional materials with hierarchical structures comprising fat or resin materials.

\section{Acknowledgment}

The present study is supported by The Cosmetology Research Foundation and Grand-in-Aid for Scientific Research on Innovation Areas (No. 16H01661) from MEXT, Japan.

\section{References}

1) Barthlott, W.; Neinhuis, C. Purity of the sacred lotus, or escape from contamination in biological surfaces. Planta 202, 1-8(1997).

2) Koch, K.; Bohn, H. F.; Barthlott, W. Hierarchically sculptured plant surfaces and superhydrophobicity. Langmuir 25, 14116-14120(2009).

3) Feng, L.; Y, Zhang.; Xi, J.; Zhu, Y.; Wang, N.; Xia, F.; Jiang, L. Petal Effect: A superhydrophobic state with high adhesive force. Langmuir 24, 4114-4119 (2008).

4) Feng, L.; Li, S.; Li, Y.; Li, H.; Zhang, L.; Zhai, J.; Song, Y.; Liu, B.; Jiang, L.; Zhu, D. Super-hydrophobic surfaces: from natural to artificial. Adv. Mater. 14, 1857-1860 (2002).

5) Sun, T.; Feng, L.; Gao, X.; Jiang, L. Bioinspired surfac- es with special wettability. Acc.Chem. Res. 38, 644652 (2005).

6) Tsujii, K.; Yamamoto, T.; Onda, T.; Shibuichi, S. Super oil-repellent surfaces. Angew. Chem. Int. Ed. Engl. 36, 1011-1012 (1997).

7) Xue, Z.; Liu, M.; Jiang, L. Recent development in polymeric superoleophobic surfaces. Polymer Physics 50, 1209-1224(2012).

8) Miyamoto, Y.; Kirihara, S.; Kanehira,S.; Takeda, M. W.; Honda, K.; Sakoda, K. Smart processing development of photonic crystals and fractals. Int. J. Appl. Ceram. Technol. 1, 40-48(2004).

9) Miyamoto, Y.; Kirihara, S.; Takeda, M.; Honda, K.; Sakota, K. Structure design for localization of electromagnetic waves in photonic fractals. Materials Integration 18, 40-45 (2005).

10) Miyamoto, Y.; Kirihara, S.; Kanaoka, H. Developments of new processing of ceramics for nanotechnology. $J$. Soc. Mater. Sci. Jpn. 55, 1051-1055(2006).

11) Parfenov, A.; Gryczynski, I.; Malicka, J.; Geddes, C.D.; Lakowicz, J.R. Enhanced fluorescence from fluorophores on fractal silver surfaces. J. Phys. Chem. B 107, 8829-8833(2003).

12) Onda, T.; Shibuichi, S.; Satoh, N.; Tsujii, K. Super-water-repellent fractal surfaces. Langmuir 12, 21252127 (1996).

13) Shibuichi, S.; Onda, T.; Satoh, N.; Tsujii, K. Super water-repellent surfaces resulting from fractal structure. J. Phys. Chem. 100, 19512-19517(1996).

14) Shibuichi, S.; Yamamoto, T.; Onda, T.; Tsujii, K. Super water-and oil-repellent surfaces resulting from fractal structure. J. Colloid Interface Sci. 208, 287-294 (1998).

15) Fang, W.; Mayama, H.; Tsujii, K. Spontaneous formation of fractal structures on triglyceride surfaces with reference to their super water-repellent properties. $J$. Phys. Chem. B 111, 564-571(2007).

16) Minami, T.; Mayama, H.; Nakamura, S.; Yokojima, S.; Shen, J. W.; Tsujii, K. Formation mechanism of fractal structures on wax surfaces with reference to their super water-repellency. Soft Matter 4, 140-144(2008).

17) Mayama, H.; Tsujii, K. Mengaer sponge-like fractal body created with a novel template method. J. Chem. Phys. 125, 124706 (2006).

18) Ono, Y.; Mayama, H.; Furo, I.; Sagidullin, A. I.; Matsusima, K.; Ura, H.; Uchiyama, T.; Tsujii, K. Characterization and structural investigation of fractal porous-silica over an extremely wide scale range of pore size. $J$. Colloid Interface Sci. 336, 215-225 (2009).

19) Tomalia, D. A.; Baker, H.; Dewald, J.; Hall, M.; Kallos, G.; Martin, S.; Roeck, J.; Ryder, J.; Smith, P. A new class of polymers: starburst-dendritic macromolecules. Polymer J. 17, 117-132(1985).

20) Esfand, R.; Tomalia, D. A. Poly (amidoamine) 
(PAMAM) dendrimers: from biomimicry to drug delivery and biomedical applications. DDT 6, 427-436 (2001).

21) Farin, D.; Avnir, D. Surface fractality of dendrimers. Angew. Chem. Int. Ed. Engle. 30, 1379-1380 (1991).

22) Shang, J.; Wang, Y.; Chen, M.; Dai, J.; Zhou, X.; Kuttner, J.; Hilt, G.; Shao, X.; Michael, J.; Wu, K. Assembling molecular Sierpiński triangle fractals. $\mathrm{Na}$ ture Chem. 7, 389-393 (2015).

23) Newkome, G.R.; Wang, P.; Moorefield, C.N.; Cho, T.J.; Mohapatra, P.P.; Li, S.; Hwang, S.; Lukoyanova, O.; Echegoyen, L.; Palagallo, J.A.; Iancu, V.; Hla, S. Nanoassembly of a fractal polymer: a molecular "Sierpinski Hexagonal Gasket”. Science 312, 1782-1785 (2006).

24) Jérémie Brunet 3D fractals, URL, http://www.shapeways.com/shops/3dfractals

25) Matsushita, M. Self-similarity, self-affinity and multifractals. Nagare 9, 145-157 (1990).

26) Petzold, R.; Zeilhofer, H.F.; Kalender, W.A. Rapid prototyping technology in medicine-basics and applica- tions. Comput. Med. Imag. Grap. 23, 277-284(1999).

27) Ryder, G.; Ion, B.; Green, G.; Harrison, D.; Wood, B. Rapid design and manufacture tools in architecture. Autom. Constr. 11, 279-290(2002).

28) Inoue, K.; Asai, S.; Sumita, M.; Inoue, K.; O-oya, S. Analysis of laces and meshes by means of fractal images and dimensions. Sen'i Gakkaishi 52, 542-552 (1996).

29) Takayasu, H. The fractal theory. J. Inst. Image Inform. TV. Engnr. 50, 686-692(1996).

30) Nonomura, Y.; Morita, Y.; Hikima, T.; Seino, E.; Chiba, S.; Mayama, H. Spreading behavior of water droplets on fractal ager gel surfaces. Langmuir 26, 16150$16154(2010)$.

31) Mandelbrot, B.B. The Fractal Geometry of Nature. Freeman, New York (1982).

32) Dillon, C.G.; Carey, P.F.; Worden, R.H. Fractscript: a macro for calculating the fractal dimension of object perimeters in images of multiple objects. Comput. Geosci. 27, 787-794(2001). 